\title{
Introduction to Peritoneal Cavity Carcinoma
}

\author{
Paris A. Kosmidis
}

The most frequent tumors in the peritoneal cavity are the metastatic. Ovarian, gastric, and colon cancers are the commonest which spread through intraperitoneal seeding. Other primary cancers, such as melanoma, lung, and breast cancers, can spread through mesenteric arteries to the peritoneum. Lymphoma may disseminate to the peritoneal cavity through lymphatic pathways [1].

CT and MRI are the most frequently used imaging methods for detecting peritoneal implants. These methods are also useful for response evaluation and follow-up. In case of increased accuracy, PET and diffuse-weighted images may be used effectively. This is particularly important for ovarian and colon cancers in which surgical interventions in cases of exclusive peritoneal disease are promising.
Pseudomyxoma peritonei is a special type of neoplasia which spread throughout the peritoneal cavity following rupture of a mucin-producing tumor of the appendix or ovary.

Primary peritoneal malignant tumors are rare and include mesothelioma, peritoneal serous carcinoma, leiomyosarcoma, and desmoplastic small round cell tumors [2].

\section{References}

1. Meyers MA (2000) Intraperitoneal spread of malignancies. In: Meyers MA (ed) Dynamic radiology of the abdomen: normal and pathologic anatomy, 5th edn. Springer, New York, pp 131-263

2. Levy A, Arnaiz J, Shaw J et al (2008) Primary peritoneal tumors: imaging features with pathologic correlation. Radiographics 28:583-607

P. A. Kosmidis

Medical Oncologist,

Head, Medical Oncology Department,

Hygeia Hospital, Athens, Greece

e-mail: parkosmi@otenet.gr 